
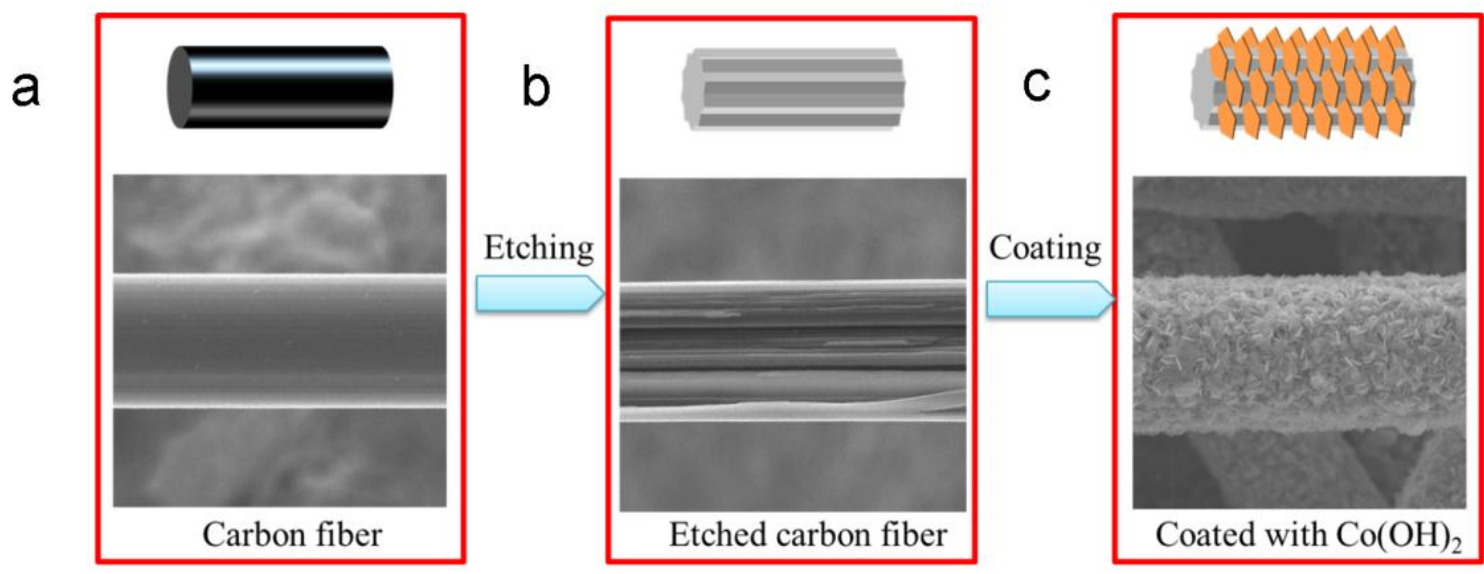


\title{
Vertically Aligned Cobalt Hydroxide Nano-Flake Coated Electro-Etched Carbon Fiber Cloth Electrodes for Supercapacitors
}

\author{
Qian Cheng, ${ }^{1,2}$ Jie Tang, ${ }^{1,2, *}$ Han Zhang, ${ }^{1}$ Lu-Chang Qin ${ }^{3}$ \\ ${ }^{1}$ National Institute for Materials Science, 1-2-1 Sengen, Tsukuba 305-0047, Japan \\ ${ }^{2}$ Doctoral Program in Materials Science and Engineering, University of Tsukuba, \\ Tsukuba 305-8577, Japan \\ ${ }^{3}$ Department of Physics and Astronomy, University of North Carolina at Chapel Hill, \\ Chapel Hill, NC 27599-3255, USA
}

\begin{abstract}
We describe preparation and characterization of nanostructured electrodes using $\mathrm{Co}(\mathrm{OH})_{2}$ nano-flakes and carbon fiber cloth for supercapacitors. Nanostructured $\mathrm{Co}(\mathrm{OH})_{2}$ flakes are produced by electrodeposition and they are coated onto the electro-etched carbon fiber cloth. A highest specific capacitance of $3404.8 \mathrm{~F} \mathrm{~g}^{-1}$ and an area-normalized specific capacitance of $3.3 \mathrm{~F} \mathrm{~cm}^{-2}$ have been obtained from such electrodes. Morphology and structure of the nanostructured electrodes were characterized have been by scanning electron microscopy (SEM) and transmission electron microscopy (TEM). The electrochemical properties have been studied by cyclic voltammetry $(\mathrm{CV})$, constant-current charge and discharge, electrochemical impedance spectroscopy (EIS), and long-time cycling.
\end{abstract}

Fax: +1-919-962-0480

Email: lcqin@unc.edu (L.-C. Qin), tang.jie@nims.go.jp (J. Tang). 


\section{Introduction}

With the rapidly growing development and applications of portable electronics, hybrid electric vehicles (HEV), and pure electric vehicles (EV), there has been an ever increasing demand of high performance energy storage devices. Electric double-layer capacitors, which are also often known as electrochemical capacitors or supercapacitors, offer a promising alternative to meet the increasing power demand of energy storage systems $[1,2]$. Supercapacitors have attracted great attention in recent years because of their pulsed high power supply, long cycle life (>100,000 cycles), simple operation mechanism, and high dynamics of charge propagation, which made supercapacitor an ideal energy storage device for applications requesting short load cycle and high reliability [3]. The materials that have been used for supercapacitor electrodes can be divided into three categories. The first category is carbon material, such as activated carbon [4,5], carbon nanotubes [6], graphene [7-9], and their composites [10]. The use of carbon material is based on the mechanism of double-layer capacitance and the capacitors store the electric charges electrostatically by invoking reversible adsorption of ions of the electrolyte onto active materials that are electrochemically stable and have high accessible surface area [11]. The second category is metal oxides. They are used in redox-based electrochemical capacitors, where transition metal oxides, such as $\mathrm{MnO}_{2}$ [12-15] and $\mathrm{RuO}_{2}$ [16,17], are used for obtaining fast and reversible redox reactions at the surfaces. The metal oxides usually have high theoretical specific capacitance but often suffer from poor power performance due to their relatively high electrical resistance. The third category is conductive polymers, such as polyaniline [18-21] and polypyrrole [22-24]. Conducting polymer such as polyaniline (PANI) has many advantageous properties including low cost, ease of synthesis, good stability in air, and high conductivity [25]. When PANI is used in supercapacitors, specific capacitance of 233-1220 F g ${ }^{-1}[9,20,26,27]$ has been achieved. However, conductive polymer-based supercapacitors usually suffer from poor stability during cycling because of destabilization of the polymer backbone.

To increase further the specific capacitance of supercapacitor electrodes, various approaches including coating metal oxides or conductive polymers onto the carbon matrix have been explored intensively recently [9,28,29]. Among all the active materials, metal oxides and metal hydroxides have been considered as the most promising electrode material for supercapacitors since they often show very high theoretical values of specific capacitance. Cobalt hydroxide $\left(\mathrm{Co}(\mathrm{OH})_{2}\right)$ is an outstanding example material of promising potential for fast insertion and desertion of electrolyte ions [30]. Its high theoretical specific capacitance of $3458 \mathrm{~F} \mathrm{~g}^{-1}$ has also made it a very attractive electrode 
material for supercapacitors [31]. However, the supercapacitor electrodes usually need metal current collectors to conduct the electrons, though the metal component would decrease greatly the weight-normalized specific capacitance of the supercapacitor device in practice. Furthermore, the area-normalized capacitance is also a very important indicator of the performance of supercapacitors in industrial applications. A lower area-normalized specific capacitance means that larger electrodes are needed to reach a certain capacitance, which in turn often leads to the use of more current collector material and lower gravimetric specific capacitance in applications [27,32-35]. An example of recent work on this material is to coat $\mathrm{Co}(\mathrm{OH})_{2}$ onto a metal foil and zeolite to explore their electrochemical performance for supercapacitor applications [36].

In this study, we fabricated vertically aligned $\mathrm{Co}(\mathrm{OH})_{2}$ nano-flakes onto electro-etched carbon fiber cloth by electrodeposition as the active material with which we have achieved a highest specific capacitance of $3405 \mathrm{~F} \mathrm{~g}^{-1}$, approaching its theoretical value of $3458 \mathrm{~F} \mathrm{~g}^{-1}$. The carbon fiber cloth was etched by electro-etching to increase the surface area of the carbon fibers and the wettability to the electrolyte. The use of carbon fiber cloth is to take advantage of its high electrical conductivity, chemical stability, three-dimensional network structure, high porosity, and low cost. It can also serve as a low-weight current collector. The benefits of choosing carbon fiber cloth as the backbone material are multifold. Firstly, the macropores in the structure of carbon fiber cloth are expected to facilitate the diffusion of electrolyte into the electrode. Secondly, the carbon fiber cloth with selective additives can also be made into flexible electrodes to produce wearable energy storage devices. Thirdly, when $\mathrm{Co}(\mathrm{OH})_{2}$ nano-flakes are directly grown onto the electro-etched carbon fiber cloth electrodes, the supercapacitor can be made binder-free to lead to smaller interfacial resistance and to enhance the electrochemical reaction rate. Most important of all, the carbon fiber cloth of high electrical conductivity can serve as the current collectors at the same time and this will improve the specific capacitance significantly.

\section{Experimental}

The carbon fiber cloth was purchased commercially (Toray, Inc., Japan) and it has a thickness of $0.19 \mathrm{~mm}$. Its lateral surface resistance is $5.8 \mathrm{~m} \Omega \cdot \mathrm{cm}$ and the perpendicular resistance is $80 \mathrm{~m} \Omega \cdot \mathrm{cm}$. The density of the carbon fiber cloth is $0.44 \mathrm{~g}$ $\mathrm{cm}^{-3}$ and the average diameter of the carbon fibers is $8 \mu \mathrm{m}$. Cobalt acetate, potassium hydroxide, and ethanol of analytical reagent grade were purchased from Sigma-Aldrich. 
Electro-etching of the carbon fiber cloth was carried out using a potentiostat operated at $2 \mathrm{~V}$ for 10-30 $\mathrm{min}$ for obtaining coatings of different thicknesses. The electro-etched carbon fiber cloth $\left(1 \times 2 \mathrm{~cm}^{2}\right)$ was used as the working electrode and a platinum plate $\left(1 \times 2 \mathrm{~cm}^{2}\right)$ was used as the counter electrode. All potential values were recorded versus the saturated $\mathrm{Ag} / \mathrm{AgCl}$ reference electrode. The distance between the working electrode and the counter electrode was fixed at $1.5 \mathrm{~cm}$. Cathodic deposition was controlled by an electrochemical station (Ivium Technologies, The Netherlands) in a $0.1 \mathrm{M}$ cobalt acetate solution. The nanostructured coating of $\mathrm{Co}(\mathrm{OH})_{2}$ was performed at constant current with the current density of $5 \mathrm{~mA} \mathrm{~cm}^{-2}$ for coating densities of 1.0, 2.0, and $3.0 \mathrm{mg} \mathrm{cm}^{-2}$ onto the electro-etched carbon fiber cloth working electrode.

The electrochemical properties and capacitance measurements of the supercapacitor electrodes were studied in a three-electrode system by cyclic voltammetry (CV), constant current charge and discharge test, and electrochemical impedance spectroscopy (EIS). The CV response of the electrodes was measured at different scan rates varying from 20 to $500 \mathrm{mV} / \mathrm{s}$. Charge and discharge testing was carried out at potentials between 0 and $0.8 \mathrm{~V}$ in $6 \mathrm{M} \mathrm{KOH}$ aqueous electrolyte solution. EIS measurements were carried out over the frequency range from $10 \mathrm{kHz}$ to $0.1 \mathrm{~Hz}$ without DC bias.

The morphology and structure of the electrode material were examined by scanning electron microscopy (SEM, JSM-6500F, JEOL) and transmission electron microscopy (TEM, JEM-2100, JEOL).

\section{Results and Discussion}

The design of our experiments is shown schematically in Fig. 1. The carbon fiber cloth (Fig. 1a) is modified first by electro-etching to obtain a highly conductive substrate with large surface area and good hydrophobicity (Fig. 1b). $\mathrm{Co}(\mathrm{OH})_{2}$ nano-flakes are then coated onto the etched carbon fiber cloth (Fig. 1c) to produce supercapacitor electrodes. This kind of electrodes is expected to possess both high weight- and area-normalized specific capacitance.

The carbon fiber cloth used in this study is made of carbon fibers which are densely packed and randomly oriented with a macroporous structure to offer better accessibility to the electrolyte. An SEM image of the pristine carbon fiber cloth is shown in Fig. 2a. In order to increase the wettability and surface area, the carbon fiber cloth is modified first by electro-etching. The inset in Fig. $2 b$ shows a carbon fiber after electro-etching. We can see clearly the etched grooves on the etched carbon fiber, which greatly increased the surface area. The electro-etched carbon fibers would also make it 
easier for the nucleation of nanomaterials. Furthermore, we also found that the electro-etched carbon fiber cloth had much better hydrophilicity than the one without etching. An SEM image of $\mathrm{Co}(\mathrm{OH})_{2}$ coated electro-etched carbon fiber cloth with coating density of $1.0 \mathrm{mg} \mathrm{cm}^{-2}$ is shown in Fig. $2 \mathrm{~b}$ in which a very uniform layer of $\mathrm{Co}(\mathrm{OH})_{2}$ coating can be seen. The thickness of the $\mathrm{Co}(\mathrm{OH})_{2}$ coating can be simply controlled by adjusting the coating time of 10,20 , and 30 minutes leading to coating densities of 1.0, 2.0, and $3.0 \mathrm{mg} \mathrm{cm}^{-2}$, respectively. Figure $2 \mathrm{c}$ shows the $\mathrm{Co}(\mathrm{OH})_{2}$ coated carbon fiber at higher magnification with a coating density of $1.0 \mathrm{mg} \mathrm{cm}$. The $\mathrm{Co}(\mathrm{OH})_{2}$ nano-flakes grown on the electro-etched carbon fiber are vertically aligned on the surface of the carbon fiber, which would offer better ion accessibility and fast ion diffusion. Figure $2 \mathrm{~d}$ is the $\mathrm{Co}(\mathrm{OH})_{2}$ coated electro-etched carbon fiber with coating density of $3.0 \mathrm{mg} \mathrm{cm}^{-2}$ and the coating becomes less uniform. Figure 2e shows a TEM image of the $\mathrm{Co}(\mathrm{OH})_{2}$ nano-flakes. Almost all the flakes have a regular hexagonal morphology. The corresponding selected-area electron diffraction (SAED) pattern (inset in Fig. 2e) showed hexagonally arranged Bragg reflections indicating that the flake is in its [001] crystallographic orientation. A representative high-resolution TEM (HRTEM) image (Fig. 2f) of the $\mathrm{Co}(\mathrm{OH})_{2}$ nano-flake shows clear lattice fringes of spacings 4.46, 2.76, and $2.37 \AA$, corresponding to the (001), (100), (101) lattice planes of the $\beta$-phase of $\mathrm{Co}(\mathrm{OH})_{2}$ (space group P3ml, lattice constant $a=3.182 \AA$ and $c=4.658 \AA$ ), respectively. Figure 3 shows the X-ray diffraction pattern obtained from the $\mathrm{Co}(\mathrm{OH})_{2}$ coated electrode material. All the sharp Bragg reflections peaks are indexed according to the structure of the $\beta$-phase of $\left(\mathrm{Co}(\mathrm{OH})_{2}\right.$.

The capacitive behavior of the electrodes was evaluated by cyclic voltammetry $(\mathrm{CV})$ as well as galvanostat charge and discharge in $6 \mathrm{M} \mathrm{KOH}$ electrolyte using a $\mathrm{Pt}$ plate as the courter electrode and $\mathrm{Hg} / \mathrm{HgO}$ as the reference electrode. Figure 4a shows the $\mathrm{CV}$ curves at different scan rates ranging from 20 to $500 \mathrm{mV} \mathrm{s}^{-1}$ in the potential range of -0.3 to $0.5 \mathrm{~V}$, which is governed by the electrochemical properties of $\mathrm{Co}(\mathrm{OH})_{2}$. Strong redox peaks and non-rectangular shape of the $\mathrm{CV}$ curves are observed as shown in Fig. 4a, indicating that the capacitive characteristics are dominated by faradic reactions and not purely electrical double-layer capacitance. The electrochemical reaction corresponding to the redox reactions can be expressed as

$$
\mathrm{Co}(\mathrm{OH})_{2}+\mathrm{OH}^{-} \Leftrightarrow \mathrm{CoOOH}+\mathrm{H}_{2} \mathrm{O}+e^{-}
$$

and the reaction at higher potential can be expressed as

$$
\mathrm{CoOOH}+\mathrm{OH}^{-} \Leftrightarrow \mathrm{CoO}_{2}+\mathrm{H}_{2} \mathrm{O}+\mathrm{e}^{-} .
$$


The charge and discharge curves of $\mathrm{Co}(\mathrm{OH})_{2}$ coated electro-etched carbon fiber cloth at different charging current of 1.0, 2.0, 3.0, and 4.0 mA are shown in Fig. 4b. The charge and discharge curves exhibited the characteristic pseudo-capacitance in all charging currents and the pseudo-capacitance is directly related to the redox peaks shown in the CV curves in Fig. 3a. The specific capacitance can be calculated from the following formula

$$
C_{m}=\frac{C}{m}=\frac{I \times \Delta t}{\Delta V \times m},
$$

where $C_{m}$ is the specific capacitance, $I$ the constant charging/discharging current, $\Delta t$ is the discharge time, $\Delta V$ is the charging potential, and $m$ is the mass of the active electrode material. Figure $4 \mathrm{c}$ is the Nyquist plot of impedance for the $\mathrm{Co}(\mathrm{OH})_{2}$ coated electro-etched carbon fiber cloth with three different coating densities. As usual, the Nyquist plot of a supercapacitor consists of three regions that are all dependent on the frequency. At high frequency, the supercapacitor behaves like a pure resistor, from which the equivalent series resistance (ESR) can be obtained from the intercept of the curve on the horizontal axis. At low frequency, the imaginary part increases sharply and a vertical and nearly vertical line is observed, indicating a capacitive behavior. In the medium frequency domain, the influence of the electrode porosities can be observed. When the frequency decreases, starting from the very high frequency, the applied electric current penetrates deeper and deeper into the porous structure of the electrode to allow more electrochemical processes to take place on more electrode surface for ion adsorption. The EIS curves given in Fig. 4c are nearly linear in the low frequency region and all have a semi-circle in high frequency region, typical for carbon-based supercapacitors. There is usually a very large circle in the activated carbon electrodes, which means large inter-granular electrical resistance between the activated carbon particles. The loop may also reveal correlations between the active material and the current collector. In the impedance of pseudocapacitors, the charge transfer resistance $R_{c t}$ can usually be correlated with the diameter of the semi-cycle observed in the Nyquist plot of the EIS data. Therefore, the EIS data shown in Fig. 4c suggests that the nanostructured $\mathrm{Co}(\mathrm{OH})_{2}$ coated electro-etched carbon fiber cloth composite has smaller charger transfer resistance and intergranular resistance [37]. The equivalent series resistance (ESR) of the supercapacitor is obtained from the intercept of the Nyquist plot with the real axis (Z1). The ESR of the three samples with increasing $\mathrm{Co}(\mathrm{OH})_{2}$ coating are 2.0, 1.8, and $1.2 \Omega$, respectively. The decreasing ESR is attributed to the increased contact area in samples with thicker coating. The ESR determines the rate that the supercapacitor can be charged and discharged and it is a very important factor to 
determine the power density of the supercapacitor.

The gravimetric specific capacitance at different coating densities was calculated from the charge-discharge curves obtained at charging current from 1 to $10 \mathrm{~mA}$, which are shown in Fig. 4d. We obtained the highest value of $3404.8 \mathrm{~F} \mathrm{~g}^{-1}$ at the charging current of $1 \mathrm{~mA}$ with coating density of $1.0 \mathrm{mg} \mathrm{cm}^{-2}$, which is quite close to the theoretical value of $3458 \mathrm{~F} \mathrm{~g}^{-1}$. The specific capacitance was $1396.1 \mathrm{~F} \mathrm{~g}^{-1}$ at charging current of $1 \mathrm{~mA}$ with coating density of $2.0 \mathrm{mg} \mathrm{cm}^{-2}$. The specific capacitance was $876.1 \mathrm{~F} \mathrm{~g}^{-1}$ at the same conditions with a coating density of $3.0 \mathrm{mg} \mathrm{cm}{ }^{-2}$. Different values of specific capacitance were also obtained at different charging current. For example, the specific capacitance was $1327.3 \mathrm{~F} \mathrm{~g}^{-1}$ at charging current of $10 \mathrm{~mA}$. At a lower value, the charging current could penetrate more into the active material and therefore the electrode can take full advantage of the redox reactions of $\mathrm{Co}(\mathrm{OH})_{2}$ with the electrolyte solution. On the other hand, as the thickness of the $\mathrm{Co}(\mathrm{OH})_{2}$ coating increases, since the electrolyte can only react with the surface atoms of the active material and the interior material with buried surfaces is all wasted practically, leading to an actual smaller specific capacitance.

The area-normalized capacitance at different charging current with the coating density of $1.0 \mathrm{mg} \mathrm{cm}^{-2}$ is shown in Fig. 4e. The largest area-normalized specific capacitance of $3.3 \mathrm{~F} \mathrm{~cm}^{-2}$ was obtained at the smallest charging current of $1 \mathrm{~mA}$. Figure 4f shows the cycling performance of the $\mathrm{Co}(\mathrm{OH})_{2}$ coated electro-etched carbon fiber cloth electrode under charging current of $10 \mathrm{~mA}$. The cycling stability shown in Fig. $4 \mathrm{f}$ is obtained with coating density of $1.0 \mathrm{mg} \mathrm{cm}^{-2}$. The capacitance dropped to $80 \%$ after 2000 cycles. It should be noted that in the first 500 cycles a larger drop in specific capacitance is usually observed and the specific capacitance becomes relatively stable after the first 500 cycles. In our case, the specific capacitance dropped about $10 \%$ in the first 500 cycles, and it dropped another $10 \%$ in the subsequent 1500 cycles. We also tested the cyclicality of larger coating densities, which exhibited almost the same behavior.

The carbon fiber cloth also served as the current collector instead of using metal current collectors. The specific capacitance is $614.0 \mathrm{~F} \mathrm{~g}^{-1}$ at $1 \mathrm{~mA}$ charging current, a high value underlining the potential for practical applications of the electrodes.

\section{Conclusions}

We have successfully fabricated vertically aligned $\mathrm{Co}(\mathrm{OH})_{2}$ nano-flakes on carbon fiber cloth by electro-deposition. The vertically aligned $\mathrm{Co}(\mathrm{OH})_{2}$ nanostructures can facilitate the accessibility of the electrolyte and can greatly increase the usage of active 
material to result in a higher specific capacitance. The use of macroporous structure of electro-etched carbon fiber cloth can further enhance the diffusion of electrolyte into the electrode to give rise to an increase of the utility ratio of active material. Moreover, the $\mathrm{Co}(\mathrm{OH})_{2}$ nano-flakes grown directly onto the electro-etched carbon fiber cloth electrode are binder free and it also helped to reduce the charge transfer resistance and ESR to $1.65 \Omega$. The electro-etched carbon fiber cloth with high conductivity can serve as the current collector to avoid using metal current collectors. As a result, highest gravimetric specific capacitance of $3404.8 \mathrm{~F} \mathrm{~g} \mathrm{~g}^{-1}$ and area-normalized specific capacitance of $3.3 \mathrm{~F}$ $\mathrm{cm}^{-2}$ have been obtained from the constructed electrodes.

These results are also useful for preparation of other metal oxide based composite electrodes targeted for energy storage applications.

\section{Acknowledgements}

This work was supported by JSPS Grants-in-Aid for Scientific Research No. 19310081 and 22310074, JST ALCA Program, and the Nanotechnology Network Project of the Ministry of Education, Culture, Sports, Science and Technology (MEXT), Japan.

\section{References}

[1] P. Simon, Y. Gogotsi, Nature Mater. 7 (2008) 845.

[2] J.R. Miller, P. Simon, Science 321 (2008) 651.

[3] L.L. Zhang, X.S. Zhao, Chem. Soc. Rev. 38 (2009) 2520.

[4] E. Frackowiak, F. Beguin, Carbon 39 (2001) 937.

[5] E. Frackowiak, F. Beguin, Carbon 40 (2002) 1775.

[6] D.N. Futaba et al., Nature Mater. 5 (2006) 987.

[7] Y. Wang et al., J. Phys. Chem. C. 113 (2009) 13103.

[8] M.D. Stoller, S.J. Park, Y.W. Zhu, J.H. An, R.S. Ruoff, Nano Lett. 8 (2008) 3498.

[9] D.W. Wang et al., ACS Nano 3 (2009) 1745.

[10] Q. Cheng, J. Tang, J. Ma, H. Zhang, N. Shinya, L.-C. Qin, Phys. Chem. Chem. Phys. 13 (2011) 17615.

[11] A.G. Pandolfo, A.F. Hollenkamp, J. Power Sources 19 (2006) 11.

[12] A.E. Fischer, K.A. Pettigrew, D.R. Rolison, R.M. Stroud, J.W. Long, Nano Lett. 7 (2007) 281.

[13] J.K. Chang, M.T. Lee, W.T. Tsai, M.J. Deng, H.F. Cheng, I.W. Sun, Langmuir 25 (2009) 11955.

[14] B. Babakhani, D.G. Ivey, J. Power Sources 195 (2010) 2110. 
[15] Q. Cheng, J. Tang, J. Ma, H. Zhang, N. Shinya, L.-C. Qin, Carbon 49 (2011) 2917.

[16] B.E. Conway, J. Electrochem. Soc. 138 (1991) 1539.

[17] J.W. Long, K.E. Swider, C.I. Merzbacher, D.R. Rolison, Langmuir 15 (1999) 780.

[18] E. Frackowiak, V. Khomenko, K. Jurewicz, K. Lota, F. Beguin, J. Power Sources 153 (2006) 413.

[19] K.S. Ryu, K.M. Kim, N.G. Park, Y.J. Park, S.H. Chang, J. Power Sources 103 (2002) 305.

[20] V. Gupta, N. Miura, J. Power Sources 157 (2006) 616.

[21] Q. Cheng, J. Tang, J. Ma, H. Zhang, N. Shinya, L.-C. Qin, J. Phys. Chem. C 115 (2011) 23584.

[22] V. Khomenko, E. Frackowiak, F. Beguin, Electrochim. Acta 50 (2005) 2499.

[23] J.H. Park, J.M. Ko, O.O. Park, D.W. Kim, J. Power Sources 105 (2002) 20.

[24] J. Li, L. Cui, X.G. Zhang, Appl. Surf. Sci. 256 (2010) 4339.

[25] J.C. Lacroix, A.F. Diaz, J. Electrochem. Soc. 135 (1988) 1457.

[26] H. Zhang, G.P. Cao, Z.Y. Wang, Y.S. Yang, Z.J. Shi, Z.N. Gu, Electrochem. Commun. 10 (2008) 1056.

[27] S.R. Sivakkumar, W.J. Kim, J.A. Choi, D.R. MacFarlane, M. Forsyth, D.W. Kim, J. Power Sources 171 (2007) 1062.

[28] J. Yan, Z. Fan, T. Wei, W. Qian, M. Zhang, F. Wei, Carbon 48 (2010) 3825.

[29] G.R. Li, Z.P. Feng, Y.N. Ou, D.C. Wu, R.W. Fu, Y.X. Tong, Langmuir 26 (2010) 2209.

[30] R.S. Jayashree, P.V. Kamath, J. Mater. Chem. 9 (1999) 961.

[31] Y.Y. Liang, L. Cao, L.B. Kong, H.L. Li, J. Power Sources 136 (2004) 197.

[32] X.F. Yu, Y.X. Li, N.F. Zhu, Q.B. Yang, K. Kalantarzadeh, Nanotechnology 18 (2007).

[33] K. Wang, J. Huang, Z. Wei, J. Phys. Chem. C 114 (2010) 8062.

[34] Y.Y. Horng, Y.C. Lu, Y.K. Hsu, C.C. Chen, L.C. Chen, K.H. Chen, J. Power Sources 195 (2010) 4418.

[35] H.L. Wang, Q.L. Hao, X.J. Yang, L.D. Lu, X. Wang, Electrochem. Commun. 11 (2009) 1158.

[36] L. Cao, F. Xu, Y.Y Liang, H.L. Li, Adv. Mater. 16 (2004) 1853.

[37] T. Zhao, H. Jiang, J. Ma, J. Power Sources 196 (2011) 860. 
1

2

3

4

5 a
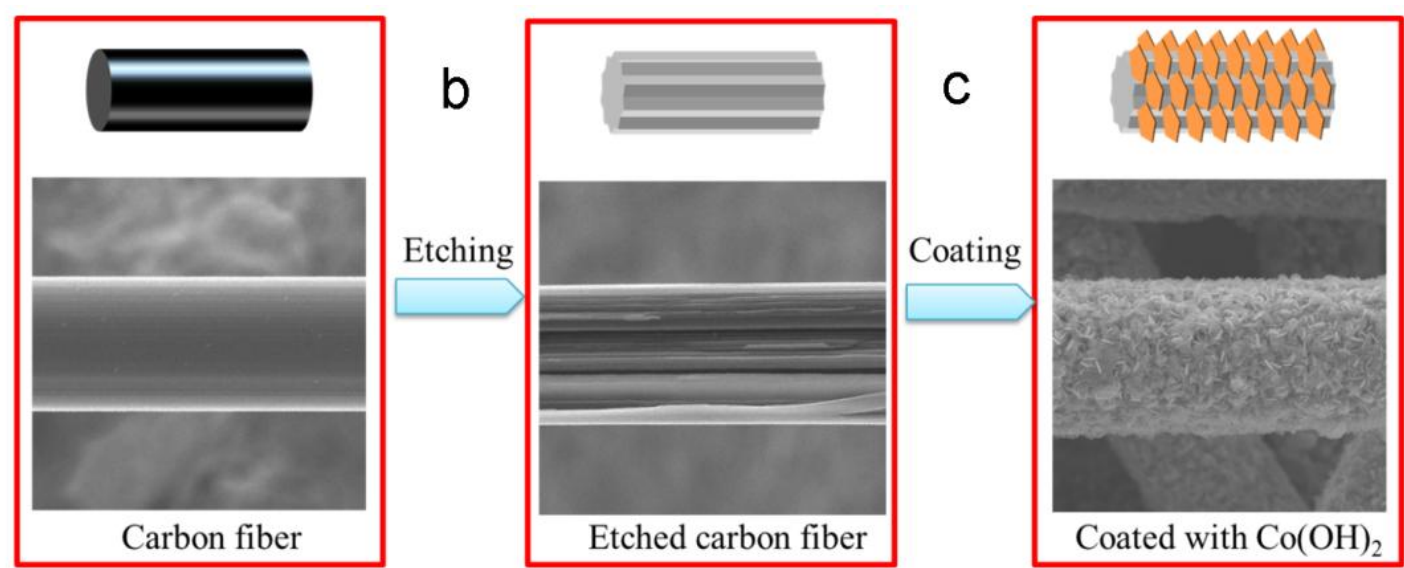

Figure 1 Schematic of making an electrode. (a) A single carbon fiber of the carbon fiber cloth without electro-etching. (b) Carbon fiber after electro-etching. (c) Electro-etched carbon fiber after electrodeposition of $\mathrm{Co}(\mathrm{OH})_{2}$ nano-flakes. 

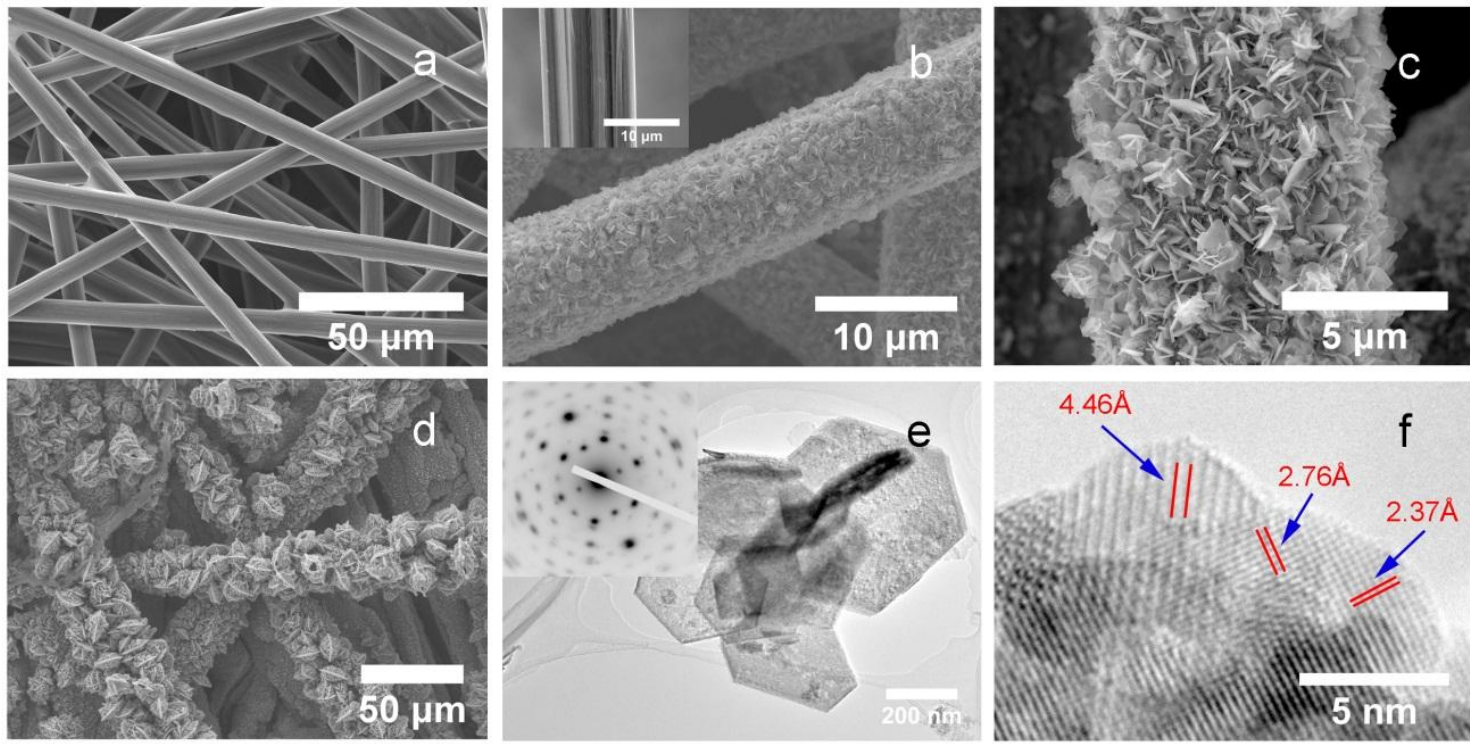

Figure 2 Morphology and structure of coated carbon fiber cloth and $\mathrm{Co}(\mathrm{OH})_{2}$. (a) SEM image of pristine carbon fiber cloth. (b) SEM image of $\mathrm{Co}(\mathrm{OH})_{2}$ coated electro-etched carbon fiber cloth. Inset is a single carbon fiber after electro-etching. (c) $\operatorname{Co}(\mathrm{OH})_{2}$ coated carbon fiber cloth with coating density of $1.0 \mathrm{mg} / \mathrm{cm}^{2}$. (d) $\mathrm{Co}(\mathrm{OH})_{2}$ coated carbon fiber cloth with coating density of $3.0 \mathrm{mg} / \mathrm{cm}^{2}$. The coating becomes less uniform compared with (c). (e) TEM image of $\mathrm{Co}(\mathrm{OH})_{2}$ nano-flakes. Inset is an electron diffraction pattern of $\mathrm{Co}(\mathrm{OH})_{2}$ in [001] orientation. (d) High-resolution TEM image of a $\mathrm{Co}(\mathrm{OH})_{2}$ nano-flake. 


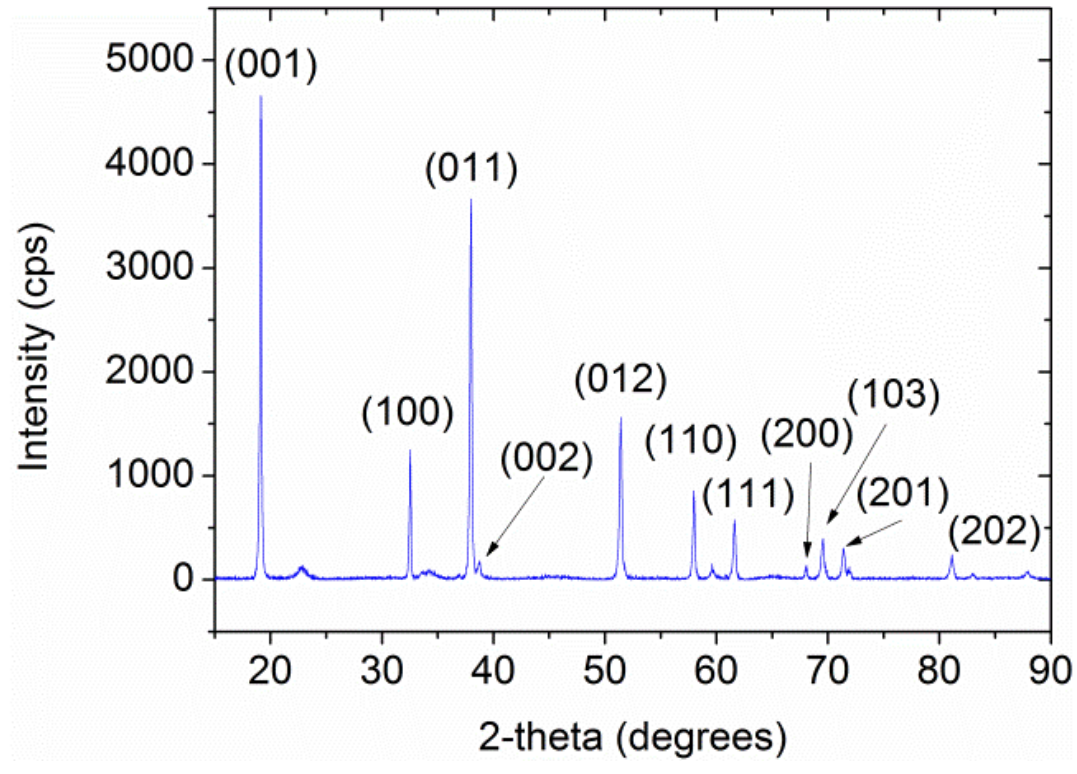

Figure 3 X-ray diffractogram of the $\mathrm{Co}(\mathrm{OH})_{2}$ electrode. All the Bragg reflections are due to the $\beta$-phase of $\mathrm{Co}(\mathrm{OH})_{2}$. 
a
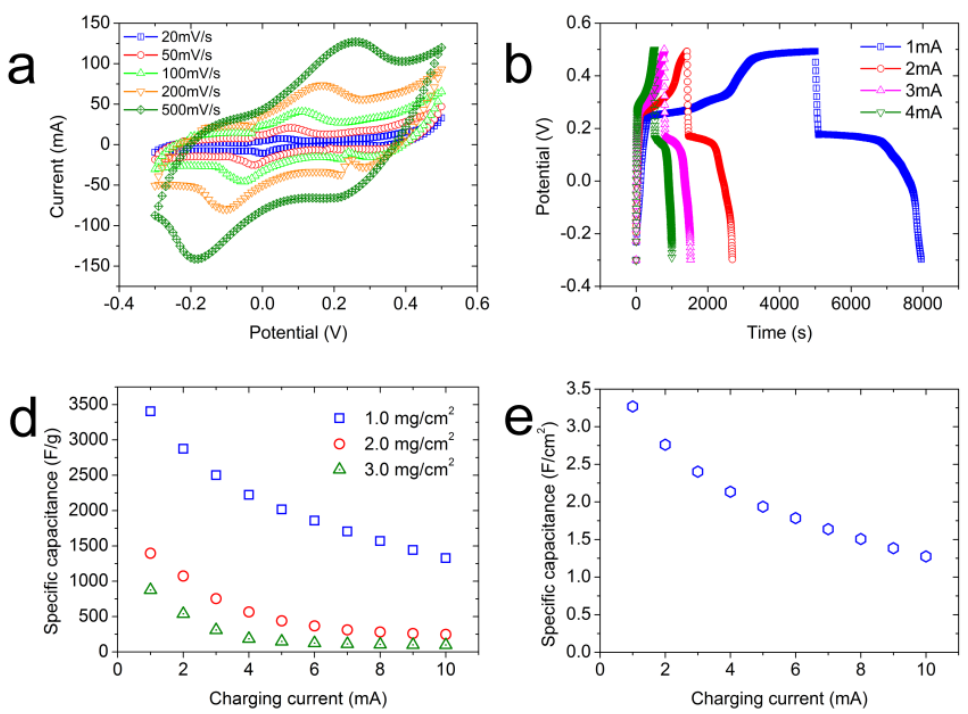
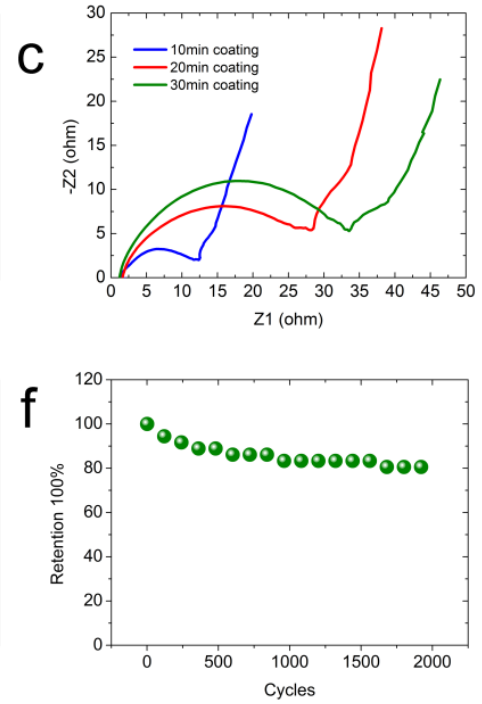

Figure 4 Electrochemical properties of $\mathrm{Co}(\mathrm{OH})_{2}$ coated carbon fiber cloth electrode. (a) $\mathrm{CV}$ curves of $\mathrm{Co}(\mathrm{OH})_{2}$ coated electrode at the scan rate of 20, 50, 100, 200, and $500 \mathrm{mV}$ $\mathrm{s}^{-1}$. (b) Charge and discharge curves of the electrode at charging current of 1, 2, 3, and 4 $\mathrm{mA}$ with coating density of $1.0 \mathrm{mg} \mathrm{cm}^{-2}$. (c) EIS curves of $\mathrm{Co}(\mathrm{OH})_{2}$ coated electrode with different coating time (10, 20, and $30 \mathrm{~min})$. (d) Mass-normalized specific capacitance at different charge currents and of different coating densities. (e) Area-normalized specific capacitance with different charging time at coating density of $1.0 \mathrm{mg} / \mathrm{cm}^{2}$. (f) Cycling stability of the $\mathrm{Co}(\mathrm{OH})_{2}$ coated electrode. 